Original Research Article

\title{
Peri-operative hyponatremia in orthopaedic injury patients
}

\author{
Channa reddy H. $^{1}$, Sharma A. ${ }^{2}$
}

${ }^{1}$ Dr. Channa reddy H, Associate Professor, ${ }^{2}$ Dr. Ambrish Sharma, Associate Professor, both authors are affiliated with Department of Orthopaedics, Basaveswara Medical College Hospital and Research Centre Chitradurga, Karnataka, India.

Address for Correspondence: Dr.Ambrish Sharma, Department of Orthopedics, Basaveswara Medical College Hospital and Research Centre, Chitradurga, Karnataka. E-mail- ambrish321@rediffmail.com

\begin{abstract}
Introduction: Hyponatremia is a common electrolyte disorder, particularly in orthopeadic trauma surgery patients and associated with adverse clinical outcomes. The aim of the study is to define the patient demographics, associated risk factors and clinical outcomes. Methods: We evaluated patients admitted with major orthopeadic trauma in a tertiary care academic institution. Elderly patients morethan 65 years, who developed hyponatremia pre-operatively or post-operatively were included in the study. Basic demographic data, risk factors for, medication use, duration of hospital stay, in patient deaths were recorded. Results: Thirty patients were included. Mean age is 72.4 years. There were 20 females (66.6\%). Risk factors (comorbidities) that cause hyponatremia were present in $73.3 \%(\mathrm{n}=22)$. The most common comorbidities are hypertension $53.3 \%(n=16)$, DM 26.6\% $(n=8)$, CRF 16.6\% $(n=5)$, heart disease 16.6\% $(n=5)$. Drug induced hyponatemia is frequently caused by thiazide diuretics $20 \%(\mathrm{n}=6)$ and anti-depressants $20 \%(\mathrm{n}=6)$. The mean serum sodium pre-operatively 132.33 $\mathrm{mmol} / \mathrm{L}$ (range 122-139) and mean post-op sodium level of $126.26 \mathrm{mmol} / \mathrm{L}$ (range 113-133) with mean post-op sodium level drop of $6.07 \mathrm{mmol} / \mathrm{L}$. Twenty one patients had mild to moderate hyponatremia. The most frequent symptom was disorientation $40 \%(n=12)$, nausea and vomiting $26.6 \%(n=8)$, headache $10 \%(n=3)$ and seventeen percent $(n=5)$ of the subjects were asymptomatic. The mean duration of hospital stay was 15.7 days (range 5-42 days), seven patients (23.3\%) required ICU care and 2 patients $(6.6 \%)$ died in the hospital. Conclusion: The most common risk factors that cause hyponatremia are hypertension and diabetes. Diuretics and antidepressant drugs are common causative factors. Average decrease in serum sodium level post operatively should be expected in this age group. Majority had mild to moderate hyponatremia. Total length of the hospital stay (TLOS) is increased compared to normal discharge. Mortality rate is also higher.
\end{abstract}

Keywords; Hyponatremia, Orthopaedic injury, Surgery, Postoperative

\section{Introduction}

Hyponatremia is the commonest electrolyte disorder observed in clinical practice. The prevalence of Hyponatremia in elderly patients with fractures vary widely from 2.8 to $26.5 \%$ at admission (pre-operative) and 2.8 to $30 \%$ develop post operatively $[1,2,3]$.

Elderly persons are more susceptible to dehydration and electrolyte disturbances. Similarly older adults are also susceptible to water retention and electrolyte abnormalities. These are exacerbated at times of physiological stress, such as in the perioperative period [4]. This predisposition of older adults to electrolyte abnormalities is further compounded by the underlying

Manuscript Received: 04 $4^{\text {th }}$ November 2017

Reviewed: $12^{\text {th }}$ November 2017

Author Corrected: $18^{\text {th }}$ November 2017

Accepted for Publication: $26^{\text {th }}$ November 2017 comorbidities that often coexist and can often be precipitated by polypharmacy. Infusion of hypotonic fluids in the peri-operative period is a well documented cause of this electrolyte imbalance [5]. Hyponatremia is associated with multiple poor clinical outcomes including falls, fractures, institutionalization, increased length of the hospital stay and mortality [6].

Many orthopeadic surgeons seems unaware of the characteristic symptoms (neurological) or the dangers of hyponatremia, predisposing already frail patients to worsened clinical outcomes. This study was undertaken to define the patient characteristics, risk factors for and clinical outcome of patients with hyponatremia with orthopaedic injuries. 


\section{Materials and Methods}

This study was conducted at the Department of Orthopaedics, Basaveswara medical college, Chitradurga, Karnataka state. It is a prospective study of elderly patients more than 65 years admitted with major bone fractures of lower limb (femur,tibia and pelvis) who developed hyponatremia (serum sodium level $<135 \mathrm{mmol} / \mathrm{l}$ ) pre or post-operatively between 2015 and 2017.

\section{Inclusion criteria:}

All these following criteria should be met to include in this study. Age more than 65 years, major bone fractures of lower limb (femur,tibia and pelvis).

Patients were included only when they have undergone surgery for stabilization for the above said fractures.Only those patients who developed hyponatremia either in the pre-operative or post-operative period or both were selected for this study.

\section{Exclusion criteria:}

Patients less than 65 years. If the said fractures were treated conservatively without surgery were not included in the study.

Patients with these fractures associated with head injury were also excluded.Patients whose serum sodium levels is normal (serum sodium level $>135 \mathrm{mmol} / \mathrm{l}$ ) both pre and post-operatively were excluded. Thirty patients $(n=30)$ were included in this study.

Basic demographic data like age, gender, site of fracture, surgical procedure underwent, medical comorbidities or pre-existing diseases were recorded.These included diseases which likely to cause hyponatremia and to estimate the total or quantitative burden of comorbidities. Medication or drug usage history likely to cause hyponatremia obtained.

Clinical outcomes- duration of hospital stay, ICU requirement, functional status, death was calculated or noted.Hyponatremia was defined as serum sodium measurement of $<135 \mathrm{mmol} / 1$.

The normal serum sodium level is $135-145 \mathrm{mmol} / \mathrm{l}$. We measured sodium levels on admission (pre-operative) and post-operatively (first 2 days). Serum sodium was measured by ion - selective electrode method (ISE).

The hyponatremia was categorized into mild hyponatremia,> 130(130-134), moderate hyponatremia $>125(125-$

129) and severe hyponatremia $<125 \mathrm{mmol} / \mathrm{l}$. In addition to the electrolytes other laboratory estimations included $\mathrm{S}$. triglycerides, S. total proteins, Random Blood sugar, BUN, Blood urea, S. Creatinine. Na level was corrected for the dilutional effect associated with hyperglycemia using the formula

Measured $\mathrm{Na}+2.4 \frac{\text { serumglucose } \mathrm{mg} / \mathrm{dl}-100}{100}$.

If any patient had symptomatic hyponatremia in the preoperative period has been treated with $0.9 \%$ normal saline till symptoms subsided and $\mathrm{Na}$ level reached near normal.

\section{Results}

The mean age of patients in the study was 72.4 years with range from $65-88$ years. Women accounted for $66.6 \%$ (n-20) of the patients.Majority of the fractures were intetrochantric fractures of the femur $63.3 \%(n=19)$.

The patient characteristics are summarized in table no1.

Some of the drugs can also cause hyponatremia. Common drugs involved in our study are thiazide diuretics $20 \%(\mathrm{n}=16)$ and antidepressant drugs $20 \%(n=6)$ of the patients.

Medications which cause hyponatremia are shown in table No.3.

Twenty-one patients had mild to moderate hyponatremia. The most common presentation was disorientation orconfusion in $40 \%(n=12)$ followed by nausea and vomiting $26.6 \%(n=8)$. Other features were headache $10 \%(n=3)$, loss of appetite $3.3 \%$ $(n=1)$, lethargy and weakness $3.3 \%(n=1)$. Seventeen percent $(n=5)$ of the patients were asymptomatic.

The mean duration of hospital stay was 15.7 days (range 5-42 days).Seven patients (23.3\%) required ICU care and two patients $(6.6 \%)$ died in the hospital. 
Table No.-1: Demographics of the study population

\begin{tabular}{|l|l|l|}
\hline \multicolumn{2}{|l|}{ No. (n=30) } & Percentage \\
\hline Age (years) & 72.4 years & \\
\hline Mean age & $65-88$ years & \\
\hline Range & \multicolumn{2}{l|}{} \\
\hline Gender & 20 & $66.6 \%$ \\
\hline Female & 10 & $33.3 \%$ \\
\hline Male & \multicolumn{2}{|l|}{} \\
\hline Fracture type & 6 & $20 \%$ \\
\hline Intra capsular & 19 & $63.3 \%$ \\
\hline Extra capsular & 5 & $16.6 \%$ \\
\hline Other fractures & 3 & $10 \%$ \\
\hline Supra condylar fracture femur & 3 & $3.3 \%$ \\
\hline Pelvis fracture & 1 & $3.3 \%$ \\
\hline Tibial plataue fracture & 1 & \\
\hline Surgery & \multicolumn{2}{l}{} \\
\hline PFN & 13 & $43.3 \%$ \\
\hline DHS & 6 & $20 \%$ \\
\hline Hemi arthroplasty & 6 & $20 \%$ \\
\hline Others & 5 & $16.6 \%$ \\
\hline Distal femur LCP & 3 & $10 \%$ \\
\hline ORIF Pelvis & 1 & $3.3 \%$ \\
\hline Prox Tibial LCP & 1 & $3.3 \%$ \\
\hline
\end{tabular}

PFN-proximal femoral nail, DHS-Dynamic hip screw, LCP- Locking compression plate, ORIF-Open reduction and internal fixation.

Table No.-2: Risk factors or comorbidities for hyponatremia

\begin{tabular}{|l|l|l|}
\hline & $\begin{array}{l}\text { Number } \\
(\mathbf{n = 3 0 )}\end{array}$ & $\mathbf{\%}$ \\
\hline Hyper tension & 16 & $53.3 \%$ \\
\hline Diabetis Mellitus & 8 & $26.6 \%$ \\
\hline Chronic kidney disease & 5 & $16.6 \%$ \\
\hline Heart disease & 5 & $16.6 \%$ \\
\hline COPD & 4 & $13.3 \%$ \\
\hline Liver disease (Cirrhosis / failure) & 3 & $10 \%$ \\
\hline Hypothyroidism & 2 & $6.6 \%$ \\
\hline $\begin{array}{l}\text { Number of comorbidities } \\
\text { Mean } \\
\text { Range }\end{array}$ & 1.43 & \\
\hline No. of patients with at least one risk factor & $0-6$ & 22 \\
\hline
\end{tabular}

Cardiac disease - Coronary artery disease, CHF-Congestive heart failure

The comorbidities which likely to cause (risk factors) hyponatremia were analyzed. Hypertension and diabetes mellitus were two major risk factors for hyponatremia found in our study $53.35 \%(n=16)$ and $26.6 \%(n=8)$ respectively.

The mean burden of comorbidities per patient is 1.43 (range 0-6). The comorbidities or risk factors associated with hyponatremia were presented in the table No.2. 
Table No.-3: Drugs associated with hyponatremia

\begin{tabular}{|l|l|l|}
\hline & $\begin{array}{l}\text { No. } \\
(\mathbf{n = 3 0})\end{array}$ & $\mathbf{\%}$ \\
\hline Diuretics-thiazide & 6 & $20 \%$ \\
\hline Anti depressents & 6 & $20 \%$ \\
\hline TCA - Amitryptaline & 1 & \\
SSRI's - Escitalopram & 3 & \\
Sertraline & 1 & \\
Atypical Anti Depressents- & & $16.6 \%$ \\
Mirtazapine & 1 & $6.6 \%$ \\
\hline ACE inhibitors and & 5 & \\
Angiotensin receptor blockers & & \\
\hline Anti psychotic drugs & 2 & \\
Butyrophenones - Haloperidol & 1 & \\
Phenothaizines & 0 & $3.3 \%$ \\
Atypical anti psychotics - clozpine & 1 & \\
\hline Anticonvulsants & & $6.6 \%$ \\
\hline Carbamazepine & 1 & $16.6 \%$ \\
\hline Anti Parkinson drugs & & \\
Leovdopa & 2 & \\
\hline Ca channel blockers and $\beta$ blockers & 5 & 2.56 \\
\hline Number of medications & $0-7$ & \\
Mean & 24 & \\
Range & & \\
\hline Percentage of patients on at least one medication & & \\
\hline
\end{tabular}

Table: No.-4: Serum sodium levels pre and post-operatively.

\begin{tabular}{|l|l|l|}
\hline $\mathbf{N}=\mathbf{3 0}$ & Pre-op Na level & Post-op Na level \\
\hline Mean & 132.33 & 126.26 \\
\hline Range & $122-139$ & $113-133$ \\
\hline Mean post-op drop of $\mathrm{Na}$ is & $6.07 \mathrm{mmol} / \mathrm{L}$ & \\
\hline
\end{tabular}

Sixteen patients (53.33\%) were hyponatremic on admission to the hospital. Twenty nine patients (96.66\%) developed hyponatremia after surgery. The pre-operative and post-operative sodium values are shown in table No.4.

Table No.-5 Post-op Na levels - according to gender and surgery type

\begin{tabular}{|l|l|l|}
\hline N=30 & Females $(\mathbf{n}=\mathbf{2 0})$ & Males $(\mathbf{n}=\mathbf{1 0})$ \\
\hline Mean & 125.2 & 128.0 \\
\hline Range & $113-132$ & $122-133$ \\
\hline & PFN $(\mathbf{n}=\mathbf{1 4})$ & $\begin{array}{l}\text { Arthroplasty group } \\
(\mathbf{n}=\mathbf{1 6})\end{array}$ \\
\hline Mean & 128.92 & 124.68 \\
\hline Range & $122-133$ & $113-130$ \\
\hline
\end{tabular}

Arthroplasty group includes - Hemiarthroplasty-6, DHS-6, LCP-4 of similar open dissection surgeries.

Serum sodium levels based on gender and surgery type is outlined in table no5.

\section{Discussion}

Hyponatremia can affect all ages but more common in older adults. Women are more affected than men as a result of smaller fluid volume and other sex related hormonal factors[7]. The incidence of hyponatremia in patients with fractures was more than double that of non fracture patients $(9.1 \%$ and $4.1 \%)$ in age more than 65 years ( $\mathrm{n}=364$ patents $\mathrm{V} / \mathrm{s} 364$ controls) [8]. 


\section{Original Research Article}

The etiology of hyponatremia is predominantly multi factorial, particularly in older people [9]. The disorder can be attributed to various causes including use of hypotonic fluids in the post-operative period, hypovolemia, acute kidney injury, syndrome of inappropriate anti-diuretic hormone secretion (SIADH), polypharmacy (medications) and various comorbidities [10].

The process of surgery itself stimulates non-osmotic secretion of anti-diuretic hormone (ADH or AVP) through stress (of surgery), (Post operative) pain, nausea, hypoxia, hypercarbia, pro-inflammatory cytokines (interleukins). Trauma and surgery, blood loss, volume depletion (decrease in the intravascular volume) in the post operative period leads to decrease in effective arterial blood volume, stimulates $\mathrm{ADH}$ secretion and water retention and hyponatremia [11].

Henrikus et alin two separate studies, reported risk factors for hyponatremia in orthopeadic surgery patients. The most common risk factors are hypertension $61 \%, 70.06 \%$, diabetes $19.23 \%, 27.5 \%$, congestive heart failure $27.5 \%$, hypothyroidism 19.6\%, COPD (asthama) 17.2\%, Chronic kidney disease $5.9 \%[3,12]$. The most frequent risk factors in our study were also hypertension $53.3 \% \quad(\mathrm{n}=16)$, diabetes $26.6 \%(\mathrm{n}=8)$ followed by chronic kidney disease (CKD) and heart disease $16.6 \%$ each.

There is a high prevalence of comorbidities and polypharmacy in elderly patients. Cumming etal in his study of elderly patients with orthopedic injuries reported that $55 \%$ (18/33) of the patients had atleast one risk factor (comorbidities) that causes hyponatremia [13]. In our study $73 \%(22 / 30)$ of the patients had at least one risk factor.

Many studies have reported association between hyponatremia and various medications, that leads to drug induced SIADH or circulating volume depletion [14]. Thiazide diuretics commonly used for the treatment of hypertension are one of the important causes of hyponatremia ranging from $23.84 \%-75 \%$ of cases $[3,13]$.

Cumming et al, in elderly patients with fragility fractures (>65) found that $73 \%$ of the patients had on at least one medication that causes hyponatremia. The use of thiazides was observed in $75.8 \%$, antidepressants $21.2 \%$, antipsychotics $1 \%$ [13]. The frequently associated drugs in our study are also similar.Thiazides (20\%) and antidepressants (20\%) being more common.

Tambee et al, found mean serum sodium levels of 134 $\mathrm{mmol} / \mathrm{l}$ and $126 \mathrm{mmol} / \mathrm{L}$ pre-and post-operatively with a mean post-operative sodium drop of $8 \mathrm{mmol} / \mathrm{L}$ in orthopedic injury patients [5]. Henrikus et al reported a mean sodium drop of $6.6+2.7 \mathrm{mmol} / \mathrm{L}$ post-operatively in similar patients [3]. Henrikus et al in another study observed mean pre and post-operative sodium levels of $138.5+3.3$ and $131+2.3$ with mean sodium drop of 7.4 $\mathrm{mmol} / \mathrm{L}[12]$. In our study mean pre and post-operative sodium levels of 132.33 and $126.26 \mathrm{mmol} / \mathrm{l}$ respectively with mean sodium drop post-operatively of $6.07 \mathrm{mmol} / \mathrm{l}$.

Consistent with previous literature majority of the patients with hyponatremia was mild to moderate degree, $70 \%$ $(\mathrm{n}=21)$.

Hyponatremia was more severe in arthroplasty surgery group compared to PFN (124.68 V/s $128.92 \mathrm{mmol} / \mathrm{L})$ possibly due to increased surgical stress through extensive muscle and soft tissue dissection [3]. Hyponatremia can present with nausea, headache, attention impairment, gait instability and falls, conditions that are often considered normal post-operative symptoms or post-anesthetic sequale.

Hyponatremia is associated with increased total length of the hospital stay (TLOS) in a variety of clinical settings including hip fractures. Previous studies in orthopeadic injury patients have documented increase in the length of the hospital stay from $13 \%-51 \%[3,13]$. In our study the increase in TLOS was 3.7 days or $31 . \%$ increase compared to normal discharge.

This delayed discharge may be due to attempts to correct hyponatremia or hyponatremia is a marker of dyshomeostasis or comorbidities which delay discharge or cause subtle cognitive and functional impairments that complicate discharge.

All types of hyponatremia has been associated with increased mortality and morbidity (peri-operative) rates[15]. In our study 2 patients (6.6\%) died in the hospital.

In summary women are more frequently affected, Hypertension, diabetes mellitus, CKD and heart disease are common risk factors for hyponatremia. Use of thiazide diuretics and anti-depressants are common causative factors. Average post-operative decrease in serum sodium level should be expected in this elderly group of patients. Majority of the patients had mild to moderate hyponatremia.

Hyponatremia is more severe in arthroplasty surgery group than less invasive surgery like PFN. Most commonly presents with disorientation, nausea, vomiting, headache and weakness. Lessthan a quarter of patients were asymptomatic. It is associated with increased length 


\section{Original Research Article}

of the hospital stay and mortality.Awareness of these risk factors and clinical manifestations results in early detection of hyponatremia and treatment and improves patients out comes.

The symptoms oforthopedic hyponatremia were not studied previously by any other authors in the literature as for as our knowledge. We could find the symptoms of hyponatremia mentioned only in review articles. We studied the clinical manifestations of hyponatremia.The most common presentation was disorientation or

confusion in $40 \%(\mathrm{n}=12)$ of patients, followed by nausea and vomiting in $26.6 \%(n=8)$. Other features were headache,loss of appetite, lethargy and weakness.We found chronic kidney disease or acute kidney injury and SSRI antidepressant drugs are equally important cause (risk factor) of hyponatremia in our study.

We found that the surgeries which require extensive muscle and soft tissue dissection (arthroplasty) leads to severe degree of hyponatremia due to increased surgical stress compared to minimally invasive surgeries (PFN).

\section{Conclusion}

To conclude we recommend the following based on findings in our study. Any elderly patient presenting with major long bone fractures of the lower limb has the potential to develop hyponatremia,should be kept in mind.If any risk factors for hyponatremia like hypertension, diabetis mellitus are present in these fracture patients-serum sodium level should be estimated as soon as the patient is admitted to the hospital and postoperatively as well.

Drugs which likely to cause hyponatremia - thiazide diuretics and SSRIS, should be stopped if possible or replaced by other drugs during the peri-operative period. Any elderly patient having symptoms like disorientation, drowsiness, nausea and vomiting in the post-operative period should be investigated for hyponatremia.

Once hyponatremia is diagnosed, regular monitoring of serum sodium level is necessary to prevent worsening of hyponatremia. Treatment should be started at the earliest to prevent the development of serious and potentially life threatening complications.

Note: Both the authors are equally involved in data collection, compilation, analysis, referencing and manuscript writing.

Conflict of interest: None declared. Funding: Nil, Permission from IRB: Yes

\section{References}

1. McPherson E, Dunsmuir RA. Hyponatremia in hip fracture patients. Scott Med J, 2002oct 47(5):115-116 DOI: $10.1177 / 003693300204700506$.

2. Beloosesky Y, Hershkovitz A, Solovey B, Salai M, Weiss A. Hip fracture post-operation dysnatremia and $\mathrm{Na}^{+}$ courses in different cognitive and functional patients groups. Arch GerontolGeriatr. 2011; 53 (2):179-182. https://doi.org/10.1016/j.archger.2010.10.014

3. Hennrikus E., Ou, G., Kinney, B., Lehman, E., Grunfeld, R., Wieler, J., Damluji, A., Davis III, C. and Mets, B. Prevalence, Timing, Causes, and outcomes of Hyponatremia in HospilizedOrthopaedic Surgery Patients. J Bone Joint Surg Am. 2015 Nov 18;97(22):1824-32. doi: 10.2106/JBJS.O.00103.

4. Desborough JP. The stress response to trauma and surgery. BrJ Anaesth,1July2000;85(1);109-117.doi: https:// doi.org/10. 1093/bja/85.1.109.

5. Tambe AA, Hill R, Livesley PJ. Post-operative hyponatraemia in orthopaedic injury. Injury. 2003 May;34 (4):253-5.

6. Soiza RI., Hoyle GE, Chua MPW. Electrolyte and salt disturbances in older people: causes ,management and implications. Rev ClinGerontol,2008 18(2):143-158.

7. Ayus CJ, ArieffAI.Brain damage and post-operative hypnatremia;the role of gender. Neurology 1996; 46(2); 323-8.

8. Sandhu HS, Gilles E., De Vita MV, Panagopoulos G, Michelis MF. Hyponatremia associated with large bone fractures in elderly patients. IntUrolNephrol, $2009,41(3)$ : 733-737Doi 10.1007/s11255-009-9585-2.

9. Clayton JA, Le Jeune IR, Hall IP. Severe hyponatremia in medical inpatients. Aetiology, assessment and outcome. QJM 2006; 99(8): 505-511.https://doi. org/10. 1093/qjmed/hcl071

10. Bagshaw S, Townsend D, McDermid R. Disorders of sodium and water balance in hospitalized patients. Can J Anesth (2009) 56(2):151-167 DOI 10.1007/s12630-0089017-2.

11. Robertson GL. Antidiuretic hormone. Normal and disordered function. Endocrinol MetabClin North Am. 2001 Sep;30(3):671-94, vii. 


\section{Original Research Article}

12. Henrikus E, Georgeson A, Ley meister K, Mucha S, Mc Gillen B, Miller R. Defining the cause of postoperative hyponatremia in the orthopeadic patient. International journal of clinical medicine 2016; 7, 668674. DOI: $10.4236 / \mathrm{ijcm} .2016 .710072$.

13. Cumming K, Hoyle GE, Hutchison JD, SoizaRL. Prevalence, incidence and etiology of hyponatremia in elderly patients with fragility fractures. PLoS One. 2014 Feb 5;9(2):e88272. doi: 10.1371/journal.pone.0088272. eCollection 2014.
14. Liamis G, Milionis H, Elisaf M. A review of drug induced hyponatremia. Am J kidney Dis. 2008; 52(1): 144-153.

DOI: http://dx.doi.org/10.1053/j.ajkd.2008.03.004

15. Waikar SS, Mount DB, Curhan GC. Mortality after hospitalization with mild, moderate, and severe hyponatremia. Am J Med. 2009 Sep;122(9):857-65. doi: 10.1016/j.amjmed.2009.01.027.

\section{How to cite this article?}

Channa reddy $\mathrm{H}$, Sharma A. Peri-operative hyponatremia in orthopaedic injury patients. Surgical Update: Int $J$ surg Orthopedics. 2017;3(4):130-136.doi:10.17511/ijoso.2017.i04.07. 